\title{
Is a Potential Therapeutic Strategy of Periodontal Intervention on Cardiovascular Disease, Yes or No?
}

\author{
Tie-Lou Chen ${ }^{1 *}$, Xinhai Zhang1, Dongsheng $\mathrm{Xu}^{2}$, Juehui $\mathrm{Li}^{3 *}$ \\ ${ }^{1}$ Department of Periodontology, Diagnosis \& Treatment Center of Stomatological Diseases of CPLA, 411 Hospital of CPLA, \\ Shanghai, China \\ ${ }^{2}$ Department of Stomatology, Baoshan District Shanghai Hospital of Integrated Traditional and Western Medicine, Shanghai, China \\ ${ }^{3}$ Department of Stomatology, Xi'an Children's Hospital, Xi'an, China \\ Email: *chentielou2010@sina.com, *13991369960@163.com
}

How to cite this paper: Chen, T.-L., Zhang, X.H., Xu, D.S. and Li, J.H. (2017) Is a Potential Therapeutic Strategy of Periodontal Intervention on Cardiovascular Disease, Yes or No? Surgical Science, 8, 52-64. http://dx.doi.org/10.4236/ss.2017.81007

Received: November 14, 2016

Accepted: January 13, 2017

Published: January 16, 2017

Copyright $\odot 2017$ by authors and Scientific Research Publishing Inc. This work is licensed under the Creative Commons Attribution International License (CC BY 4.0).

http://creativecommons.org/licenses/by/4.0/ (c) (i) Open Access

\begin{abstract}
Aims: Cardiovascular disease (CVD) and periodontitis are both chronic inflammatory disorders which are highly prevalent in populations. Bacteria involved in the periodontal disease have been found to be cardiovascular risk markers. Periodontal pathogens may contribute to the atheroma pathogenesis. Severe periodontitis is correlated with the prevalence of bacteraemia, and poor periodontal status is an important risk factor for CVD. However, the association is unclear. If the association is causal, the periodontal therapy will lead to an attenuation of the effects on CVD. The study aimed to study if the periodontal intervention therapy presented therapeutic effects on CVD. Methods: English language literature on periodontal intervention therapy on CVD is causal or not. The literature revealed 62 papers associated with this study to investigate the relationship between periodontitis and CVD. Results: The literature supported the idea that periodontal infections had been associated with CVD. Certain periodontal therapy is associated with bacteraemia, and the prevalence of bacteraemia may arise from periodontal therapy and poor oral hygiene practices. Periodontal therapy not only presented therapeutic effects by reducing cytokine activity and C-response protein (CRP), but also caused bacteraemia transitorily. Effective antibiotic prophylaxis pre- or post-periodontal therapy presented some beneficial effects on bacteraemia or CVD. Conclusion: Severe periodontitis causes systemic inflammation and endothelial dysfunction, and goes beyond the oral cavity. Periodontal intervention would contribute to the prevention of atherosclerosis, and antibiotic prophylaxis would be helpful to decrease bacteraemia and reduce the onset of CVD.
\end{abstract}

\section{Keywords}

Periodontal Disease, Cardiovascular Disease, Periodontal Intervention, Antibiotic Prophylaxis, Yes or No 


\section{Introduction}

Periodontitis has been associated with an increased risk of cardiovascular events. Cardiovascular diseases (CVD) are the most prevalent diseases in developed countries. Disorders of blood vessels, hypertension and arteriosclerosis are the chief causes of death in the Western world, and atherosclerosis is the main cause of CVD [1]. Inflammation has emerged as a causative factor in all stages of CVD. Mortality from CVD is expected to increase by $25 \%$ in the next two decades [2]. Periodontal disease may contribute to atherogenesis and/or thromboembolic events [3]. The majority of clinical and epidemiological findings confirm the presence of an intimately association between periodontitis and CVD [4], and the prevalence and incidence of CVD are significantly increased in periodontal infection patients [5]. There is increasing evidence that chronic infections, such as periodontal diseases, could play a role in the initiation and development of coronary artery disease [6]. Numerous longitudinal epidemiological and cross-sectional studies have provided powerful evidence that there are significant associations between periodontitis and elevated risk for CVD [7] [8]. Data have shown a consistent relationship between pocket depth and incident myocardial infarction [9]. Periodontal bleeding per se is a poor indicator of odontogenic bacteraemia and gingivitis is correlated positively with the prevalence of bacteraemia [10]. Recently, attention has been focused not simply on how systematic diseases influence oral health but on how oral health may have effects that extend beyond the oral cavity. For example, a meta-analysis suggested that individuals suffering from periodontitis may have 1.14 2.2 times greater risk of developing coronary heart disease compared with individuals without periodontitis [11]. In addition, experimental evidence has showed that periodontal pathogenic bacteria, mainly Porphyromonas gingivalis, play a role in atherogenesis [12]. Several reviews have described the relationship between periodontal periodontal disease and risk of coronary heart disease or other CVD, and have suggested that periodontitis may contribute to CVD in susceptible subjects [13] [14].

Recent studies have focused on the systemic effects of periodontal intervention on indicators of CVD. Periodontal therapy has had some success in the modification of many of inflammatory indicators in systemically healthy adults [15]. Thus we must make sure if periodontal intervention could lower the risk of cardiovascular events or even prevent onset and progression of the disease. It's reported that poor oral health, especially poor periodontal status, is an important risk factor for CVD. Certain periodontal procedures are associated with bacteraemia. The prevalence of bacteraemia arise from periodontal therapy and various oral hygiene practices. It has been suggested that oral hygiene practices are responsible for spontaneous bacteraemia. Such bacteraemia is of low grade intensity and of short duration [16]. The studies have shown there is link between dental procedures and infective endocarditis [17]. Penicillin is proved to reduce bacteraemia by $84 \%-86 \%$ at $5 \mathrm{~min}$ and $95 \%-97 \%$ at $30 \mathrm{~min}$ after bacteraemia induction. These findings can be compared with a reduction of $24 \%-42 \%$ and $49 \%-76 \%$, respectively, when no prophylaxis is used. By contrast, antibiotic prophylaxis, such as single doses of $2 \mathrm{~g}$ penicillin and $3 \mathrm{~g}$ amoxicillin fail to reduce bacteraemia after dental extractions. The evidence that antiseptic mouthwashes such as chlorhexidine and povidone-iodine used prior to dental procedures may reduce the 
prevalence of bacteraemia [18]. Incidence of bacteraemia arising after scaling and root planing is $8 \%-80 \%$, and that of gingivectomy is $83 \%$, and that of flap procedure is $36 \%$ $88 \%$. Incidence of bacteraemia arising from extraction is $51 \%-100 \%$ [19].

Whether spontaneous periodontal disease induced bacteraemia or periodontal intervention procedure induced bacteraemia are still unclear. Whether periodontal intervention can be used as a potential therapeutic strategy to CVD is unknown. Therefore, we write this paper to study if periodontal therapy could reduce inflammation to decrease morbidity, and effective antibiotic prophylaxis pre- or post-periodontal therapy to reduce bacteraemia transitorily. This study focuses on the effects of periodontal therapy and antibiotic prophylaxis on CVD.

Periodontal disease is considered a potential risk factor for CVD, and it should be influential in the diagnosis and treatment of CVD patients. Increased inflammatory cytokines and CRP levels may contribute to inflammatory events, including CVD and periodontitis. Periodontal intervention may reduce CVD by inflammatory response regulation of cytokine and CRP levels. But some periodontal procedures may induce bacteraemia temporarily. Periodontal diseases can be treated effectively by periodontal intervention and preventing bacteria from entering the blood stream leading to the distant organ infection, and reducing the inflammatory cytokine (IL-6, TNF- $\alpha$ ) and CRP levels in serum, and would likely decrease the incidence of both cardiovascular and periodontal diseases. Some effective antibiotic prophylaxis pre- or post-periodontal therapy could prevent bacteraemia transitorily and prevent the onset of CVD. This paper found that periodontal intervention can reduce the incidence rate and severity of CVD. Antibiotic prophylaxis can improve the therapeutic effects and reduce the incidence of atherosclerosis. Exploration of evidence-based periodontal intervention strategy would help clinicians in planning periodontal therapy to reduce the incidence of atherosclerosis and CVD.

\section{Relevant Bacterial Evidence between Periodontal Disease and CVD (Table 1)}

Bacteria of periodontal infection are associated with the coronary vascular disease. Oral microorganisms including periodontal bacterial pathogens enter into the blood stream and form transient bacteraemia. Periodontal pathogens are present in atherosclerotic plaques where, like other infectious microorganisms such as Chlamydia pneumoniae, they may play a role in the development of CVD. Periodontal pathogen, such as $A g$ gregatibacter actinomycetemcomitans $(\mathrm{Aa})$, deteriorates ventricular remodeling after myocardial infarction in Mice [20]. Oral bacteria and bacterial products enter into the blood stream and are thought to be one of the key initiators of biological events linking oral infections to atherosclerotic vascular disease [21]. Increasing risk for systemic disease in subjects suffering from periodontal disease could be an increased prevalence and severity because of bacteraemia associated with oral bacteria [22]. Periodontal bacteria occasionally have been a correlative causative agent for infections at distant organs [23]. Studies have thus evaluated if periodontal bacteria are detectable and culturable from atherothrombotic plaques or vascular biopsies. Bacterial DNA from several periodontal pathogens has been detected in human arterectomy specimens [24]. Experimental studies suggest low concentrations of lipopolysaccharides from Porphyromonas 
Table 1. Relevant bacterial evidence between periodontal disease and CVD.

\begin{tabular}{llll}
\hline Author(s) & Study design & Outcomes & Conclusions \\
year $[$ Ref $]$ & &
\end{tabular}

Hanatani, Animal study.

T., et al., $\quad 8$ male mouse injected $\mathrm{Aa}, 6$ cases injected

2012 [20] PBS. Experimental MI induced by permanent ligation of the left anterior descending coronary artery

Iwai, T., Mini review.

2009 [21] The Medline and Tokyo Medical and Dental University databases were searched to identify the literature currently available on oral bacteria and vascular diseases.

Haraszthy, Clinical study.

V.I., et al., 50 specimens obtained, examined Cp,

2000 [22]. HCMV, and bacterial 16S ribosomal RNA

using PCR. 100 ng of chromosomal DNA extracted from specimen. PCR product generated with eubacterial primers transferred and probed with digoxigenin labeled synthetic oligonucleotides for $\mathrm{Aa}, \mathrm{Bf}$, $\mathrm{Pg}$, and $\mathrm{Pi}$.

Kebschull, Critical review.

M., et al., (1) Observational studies; (2) interventional 2010 [23] studies; (3) Potential mechanisms linking periodontal infections and atherosclerosis. (4) Periodontal pathogens in animal models of atherogenesis. (5) Available mechanistic studies.

Gaetti-Jard Clinical study.

im, E., et 44 patients displaying CVD were submitted

al., 2009 and endarterectomy of coronary arteries. 60 -

[24] $100 \mathrm{mg}$ atherosclerotic tissue removed.

Quantitative detection of periodontopathic

bacteria using TaqMan probe. Aa, Fn, Pg, Pi,

$\mathrm{Pn}$ and $\mathrm{Tf}$ determined by real-time PCR.

Mattila, K., Clinical study.

et al., 1989100 patients with acute MI and 102 controls [25] selected from community at random. Serum total cholesterol, triglyceride, and high density lipoprotein cholesterol measured on admission and 4 weeks. Lipid values obtained during second visit. Dental scores measured. The index ranged from 0 to 10 .
Plasma level of anti-Aa antibody higher in Aa-infected mice than control mice. 7 days after myocardial ischemia, Aa-positive MI hearts showed larger infarct size and length than control mice. Aa-positive MI hearts showed more MOMA-2 positive myocardial infiltrating cells compared to negative MI.

Bacteria from mouth to the bloodstream and then to vein of venous angle near supraclavicular area. Periodontal bacteria, such as Pg, activate platelets and make them mass, including bacteria without phagocytosis. Animal study showed the formation of a small arterial thrombus after continuous intravenous infusion of Pg for 2 - 4 week.

$80 \%$ of the specimens were positive in 1 or more of PCR assays. $38 \%$ were positive for HCMV and $18 \%$ positive for $\mathrm{Cp}$. The presence of bacteria in $72 \%$ of surgical specimens. $44 \%$ of the 50 atheromas were positive for at least one of periodontal pathogens. $30 \%$ of surgical specimens were positive for $\mathrm{Bf}, 26 \%$ positive for $\mathrm{Pg}, 18 \%$ positive for Aa, and $14 \%$ positive for Pi. 13 (59\%) of the 22 periodontal pathogen-positive surgical specimens were positive for 2 or more of the target species

Periodontal treatment resulted in favorable effects on subclinical markers of atherosclerosis. In vitro and in vivo studies established plausibility of a link between periodontal infections and atherogenesis. Utilized models are mostly mono-infections of host cells, and may not adequately portray human periodontitis as a polymicrobial, biofilm-mediated disease.

Total bacterial and periodontopathic bacterial DNA found in $94.9 \%$ and $92.3 \%$ respectively, of atheromatous plaques from periodontitis patients, and in $80.0 \%$ and $20.0 \%$, of atherosclerotic tissues from periodontally healthy subjects. DNA represented $47.3 \%$ of total bacterial DNA obtained from periodontitis patients. Pg, $\mathrm{Aa}$ and Pi detected most often.

Smoking was more common among acute MI, and had lower concentrations of high density lipoprotein cholesterol and higher serum concentrations of triglycerides and $\mathrm{C}$ peptide than the controls. Hypertension was more common among patients than controls. Serum total cholesterol concentration was not associated with MI. Total dental index and the pantomography index highly correlated. Two indexes higher among patients than controls.
Periodontal pathogen might deteriorate ventricular remodeling after MI through inflammatory cell infiltration

Periodontal bacteria, may play an important role in the development of various vascular diseases, such as atherosclerosis and varicose veins, through bacteremia.

Periodontal pathogens present in atherosclerotic Plaques, such as $\mathrm{Cp}$. They play a key role in the development of atherosclerosis leading to coronary vascular disease.

Periodontal therapy results in lower levels of systemic inflammation and favorable effects on subclinical markers of atherosclerosis.

Periodontopathic bacteria in coronary lesions are not coincidental and that they may contribute to the development of vascular diseases.

Dental health was significantly worse in patients with acute MI than in controls. Periodontal disease and dental caries is more common among patients with acute MI than controls

\footnotetext{
Abbreviations: Aa, Aggregatibacter actinomycetemcomitans, Pg, Porphyromonas gingivalis, Cp, Chlamydia pneumoniae; Bf, Bacteroides forsythus, Pi, Prevotella intermedia; Fn, Fusobacterium nucleatum; Pn, Prevotella nigrescens; Tf, Tannerella forsythia; HCMV, human cytomegalovirus; MI, myocardial infarction; MOMA-2, Monocyte/Macrophage marker; PCR, polymerase chain reaction; CVD, cardiovascular disease.
} 
gingivalis and Prevotella intermedia profoundly stimulate the secretion of interleukins by human macrophages. Periodontal pathogens may increase the risk of CVD through mechanisms such as platelet activation and aggregation [25].

\section{Effects of Periodontal Intervention on Inflammatory Cytokines}

Inflammatory cytokines include pro-inflammatory mediators, such as tumour necrosis factor (TNF)- $\alpha$, interleukin (IL)-1 $\beta$, IL-6 and IL-12, chemokines (e.g. IL-8), and anti-inflammatory mediators (e.g. IL-10, IL-1 antagonist and IL-4), play distinct biological effects [26]. The chemokine mainly attracts and activates neutrophils, and is considered important mediators for granulocyte accumulation. IL- 6 secreted by various cell types, such as fibroblast cells, monocyte macrophage cells, and epithelium cells, and its level is increased by bacterial lipopolysaccharide stimulated. IL- 6 can promote coagulation and result in the development of atherosclerosis. The enhancement of IL-6 levels has been associated with both cardiovascular-risk and periodontal disease [27]. TNF- $\alpha$ stimulates the production of chemokines and cytokines, collagenase, prostaglandin E2 [28], cellular adhesion molecules and bone resorption-related factors. Based on the biological properties of pro-inflammatory cytokines, high levels of TNF- $\alpha$ and IL-6 in plasma have been associated with an increased morbidity and mortality of cardiovascular events [29]. Peripheral blood mononuclear cells from periodontitis subjects released higher levels of TNF- $\alpha$ and IL- 6 than those from healthy subjects. In contrast, mononuclear cells obtained from healthy subjects presented higher amounts of IL-8 than those from periodontitis individuals [30]. In a prospective study of apparently healthy men, the IL-6 levels of those who subsequently had a myocardial infarction were higher than matched control without myocardial infarction during a 6-year follow-up [31]. IL-6 levels may be a predictor of risk of future myocardial infarction in apparently healthy men. Severe periodontitis can result in systemic inflammation characterized by elevated serum IL-6 [32]. A recent study reported that subjects with both coronary artery disease and periodontitis had significantly higher serum IL-6 concentrations compared with subjects with coronary artery disease who had no periodontitis [33]. TNF- $\alpha$ is involved in the initiation and development of coronary artery disease [34], and its levels increased in patients with periodontitis [35]. Conversely, IL-10 suppresses the inflammation reaction and minimizes tissue destruction [36].

Periodontal therapy can reduce the levels of pro-inflammory cytokines at three months post-therapy, and thus may lower the CVD incidence. In addition, the current study concluded that periodontal therapy had minimal impact on levels of TNF- $\alpha$ and IL-1 $\beta$ [37]. The effects of periodontal intervention on serum IL-6 are not consistent. Some studies showed that periodontal therapy results in a decrease in IL-6 levels [38], although others found no significant differences post-therapy [39], In a recent study on coronary artery disease patients with periodontitis, periodontal therapy reduced serum concentrations of IL-6 at 6 months post-therapy [33]. Similar findings can be obtained in which periodontitis patients with coronary heart disease received mechanical periodontal therapy and control patients did not receive periodontal therapy. The serum IL-6 levels in periodontal therapeutic group decreased significantly than those in the control group at 3 months after periodontal therapy. The effect of periodontal therapy 
on circulating TNF- $\alpha$ levels is not consistent. An investigation showed that the TNF- $\alpha$ levels decreased significantly after periodontal therapy [40]. And others reported that no effect could be seen following periodontal intervention [41].

\section{Effect of Periodontal Intervention on C-Reactive Protein}

$\mathrm{CRP}$, an acute phase reactant protein, is responsible for the increase in the vascular inflammation. Its main function is thought to result in the activation of the complement system. In humans, the levels of CRP in plasma rise rapidly (as much as 1000 folds or more) after an acute inflammatory stimulus. This increase is mainly due to increased synthesis of this protein by hepatocytes that are stimulated by various cytokines, particularly IL-6 [42]. CRP level measurement can provide a more sensitive means of detecting signs of inflammation and trauma. CRP concentration in serum is significantly increased in patients with coronary heart disease and myocardial infarction. Elevated plasma levels of CRP have been documented as a major risk factor for CVD, and may be used as an important indication of coronary artery disease and acute myocardial infarction [43]. In a cohort study, it was noted that increasing levels of serum high-sensitivity CRP were associated with the risk of cardiovascular events, and that CRP was the strongest univariate predictor of the risk of such events [44]. CRP can increase low density lipoprotein uptake into macrophages and inhibit endothelial nitric-oxide synthase expression in aortic endothelial cells. CRP can also induce adhesion molecule expression in human endothelial cells in serum and may contribute to the hypercoagulative state in coronary disease patients [45]. Recently, accumulated evidence has demonstrated the association between periodontitis and CRP. The serum CRP is increased in systemically healthy subjects with periodontitis [46]. It was reported that subjects with periodontitis had $1.65 \mathrm{mg} / \mathrm{L}$ higher serum CRP compared with individuals without periodontitis [47]. Studies have showed that periodontitis patients with CVD or hypertension had significantly higher serum sensitivity CRP concentrations than patients without periodontitis [48]. Periodontal diseases are associated with changes in serum components that are consistent with the increased circulation of IL-6 and elevated levels of high sensitivity CRP [49]. Recently, clinical trials have shown a significant reduction of CPR levels and other inflammatory markers in serum after periodontal therapy [50]. Furthermore, a multi-centered randomized control study showed that periodontal therapy can reduce the CRP levels from high to moderate in non-obese periodontal disease patients [51]. Similar results confirmed by a randomized controlled trial that the levels of CRP reduced after periodontal therapy [52]. Periodontal intervention and periodontal maintenance may contribute to the systemic inflammation and thereby decrease the incidence and morbidity of CVD [53]. But other studies have found that periodontal intervention, especially one that involves periodontal surgery and dental extraction, will increase the levels of inflammatory mediators immediately [54]. Therefore, full-mouth disinfection agent has been introduced as a short-term method to suppress periodontal pathogens within $24 \mathrm{~h}$ [55].

\section{Effects of Periodontal Procedures on CVD and Antibiotic Prophylaxis (Table 2)}

Gingival inflammation is correlated positively with the prevalence of bacteraemia [11]. 
Table 2. Effects of periodontal procedures on CVD and antibiotic prophylaxis.

\begin{tabular}{ll}
\hline Author(s) & Study design
\end{tabular}

Bahekar, Systematic review.

A.A., et Literature revealed 5 prospective cohort

al., 2007 studies, 5 case control studies, 5

[11] cross-sectional studies. Individual studies were adjusted for confounding factors such as age, sex, diabetes mellitus, and smoking.

Ioannidou, Review.

E., et al., English literature of periodontal treatment

2006 [15] on CRP levels assessed, at least 2 months after periodontal treatment. The search conducted in MEDLINE between 1966 and July 2005 and Cochrane Central Register of Controlled Trials.

Seymour, Review.

R.A., et al., The paper reviewed the effect of 2003 [19] cardiovascular drugs on the periodontium and management of patients with periodontal diseases. The risk of infective endocarditis arising from periodontal procedures, and the inter-relationship between periodontal disease and CAD.

Hokamura, Minireview.

K., et al., Clarify the involvement of Pg on the

2010 [56] mechanisms of development of aortic intimal hyperplasia. Intravenous administration of Pg induced intimal hyperplasia in the mouse model with photochemical impairment of the femoral artery.

Hall, G., Clinical study.

et al., 199360 healthy patients randomized to receive [57] placebo, penicillin-V (2 g), or amoxicillin 1 hour before dental extraction was performed. Blood samples for microbiological investigation collected before, during, and 10 minutes post surgery and processed by lysis filtration

Maharaj, Clinical study.

B., et al., 160 patients for antimicrobial prophylaxis 2012 [59] before dental extraction. Group A served as control, group B rinsed with chlorhexidene, group $\mathrm{C}$ took $3 \mathrm{~g}$ amoxicillin orally, group $\mathrm{D}$ took $600 \mathrm{mg}$ clindamycin.
Prospective cohort studies indicated that individual with PD had a 1.14 times higher risk of developing CHD than controls. Case control studies showed great risk of developing CHD. Prevalence of CHD in cross-sectional studies significantly greater among individual with $\mathrm{PD}$ than in those without PD. Cohort studies showed 1.24 times increase risk of development of CHD in patients with $<10$ teeth.

Literature yielded 814 citations of which 10 met the inclusion criteria. Meta-analysis of RCT included that the difference in serum CRP levels is not significantly different between the two arms. The single-cohort studies suggested that the difference on serum CRP levels was not significantly different before and after treatment.

Stopping anticoagulant therapy prior to periodontal procedures is putting patients at a greater risk of thromboembolic disorders compared to the risk of prolonged bleeding. Spontaneous bacteraemia arising from a patient's oral hygiene practices is more likely to be the cause of endocarditis than one-off periodontal procedures. The risk of death from penicillin appears to be greater than the risk of death arising from infective endocarditis.

No changes identified in the mice without aortic impairment, even with Pg infection. S100A9 and the SMemb were significantly over expressed on surfaces of smooth muscle cells present in injured blood vessels. Increased expressions of S100A9 and SMemb proteins observed in aneurismal specimens obtained from Pg infected patients. Bacteremia induced by Pg leads to intimal hyperplasia associated with over expressions of S100A9 and SMemb.

The overall incidence of bacteremia after dental extraction was $95 \%, 90 \%$, and $85 \%$, respectively, for the 3 groups. For $>90 \%$ of 126 strains of viridans streptococci tested, the MIC of penicillin-V and ampicillin were $\leq 0.125 \mathrm{mg} / \mathrm{L}$

The proportion of patients who had post-extraction bacteraemia in groups A, B, C and D was $35 \%, 40 \%$, $7.5 \%$ and $20 \%$, respectively. The differences between the control and amoxicillin groups and between the chlorhexidine and amoxicillin groups were statistically significant.
Both the prevalence and incidence of CHD were significantly increased in PD. The PD must be a risk factor for CHD. Prospective studies required to prove the risk reduction with the treatment of PD.

Evidence indicated that systemic inflammation presented in patients with periodontal disease. RCT and single cohort studies do not support that periodontal treatment can reduce CRP levels.

CAD can interact with the periodontium. Further investigation must Manage anticoagulant therapy and the risk from infective endocarditis.

$\mathrm{Pg}$ is a causative event in the development of aortic hyperplasia in periodontitis. Upregulation of the S100A9 by $\mathrm{Pg}$ is important event in development of intimal hyperplasia in aorta.

The protective effect of prophylactically administered penicillins must be due to interference with the steps in the development of endocarditis, other than the transient bacteremia that occurs initially.

None of the treatments prevented post-extraction bacteraemia and confirmed earlier reports that bacteraemia is not completely eliminated by antibiotics.

Abbreviations: CHD, coronary heart disease; PD, peridontal pocket depth; CRP, C-reactive protein; RCT, randomized controlled trials; CAD, coronary artery disease; Pg, Porphyromonas gingivalis, S100A9, S100 calcium-binding protein A9; SMemb, embryonic isoform of myosin heavy chain; MIC, minimal inhibitory concentration. 
Periodontal bleeding per se is a poor indicator of odontogenic bacteraemia. The certain periodontal procedures are associated with bacteraemia. The prevalence of bacteraemia arise from periodontal therapy and various oral hygiene practices. In many instances the prevalence of bacteraemia after such innocuous events is comparable with that occurring after periodontal procedures. It has been suggested that oral hygiene practices are responsible for spontaneous cases of bacteraemia. Such bacteraemias either from periodontal procedures or oral hygiene practices are of low-grade intensity (10 - 200 $\mathrm{CFU} / \mathrm{ml}$ of blood) and of short duration [15]. Bacteremia induced by Porphyromonas gingivalis ( $\mathrm{Pg}$ ) leads to intimal hyperplasia associated with overexpressions of embryonic isoform of myosin heavy chain and oral hematogenous spreading of Pg is a causative event in the development of aortic hyperplasia in periodontitis patients [56]. Penicillin has been shown to reduce bacteraemia by $84 \%-86 \%$ at $5 \mathrm{~min}$ and $95 \%-97 \%$ at $30 \mathrm{~min}$ after bacteraemia induction. These results can be compared with a reduction of $24 \%-42 \%$ and $49 \%-76 \%$, respectively, when no prophylaxis used [19]. By contrast, other workers have shown that single doses of $2 \mathrm{~g}$ penicillin and $3 \mathrm{~g}$ amoxicillin fail to reduce bacteraemia after dental extractions [57]. Although most attention has been focused on antibiotic prophylaxis, there is evidence that antiseptic mouthwashes such as chlorhexidine and povidone-iodine used prior to certain periodontal procedures may reduce the prevalence of bacteraemia [58]. Maharaj B [59] found that there were none of the significant treatments prevented post-extraction bacteraemia and confirmed earlier reports that bacteraemia was not completely eliminated by antibiotics. Recently, studies have showed that there is no obvious increase in the incidence of infective endocarditis cases or deaths in the two years after the guideline was introduced about the cessation prescribing antibiotic prophylaxis. The findings support the cessation of prescribing antibiotic prophylaxis [60]. Therefore further clinical trials should determine if periodontal intervention combined with drug therapy would reduce the incidence of CVD.

\section{Conclusion}

Periodontal inflammatory response could exacerbate vascular inflammation via secreted cytokines that ultimately modulate atherosclerosis and CVD. The inflammatory cytokine IL- 6 and TNF- $\alpha$, and CRP levels in serum increased are association with CVD and periodontal diseases. Periodontal intervention had a positive impact on the established risk factors for CVD to reduce inflammatory responses. Periodontal intervention studies have strengthened the evidence for an association between the periodontal disease and CVD, and have also indicated a causal link. As some periodontal procedures may increase bacteraemia temporarily, effective antibiotic prophylaxis is necessary to the prevention bacteraemia and the onset of CVD [61]. However, detailed mechanism of spontaneous periodontal disease induces bacteraemia and the influence of periodontal intervention procedures inducing bacteraemia leading to CVD is unknown. Because of these different research results, carefully designed randomized trials based on this concept with longer follow-up and clinical observation are required to prove if periodontal intervention combined with drug therapy would reduce the incidence of CVD. 


\section{Acknowledgements}

This study was supported by Shanghai Health and Family Planning Commission Research Grants (No. 20134418 and General Logistics Department Research Grants of CPLA (No. CHJ13J035). The study was also supported by Shanghai Municipal Hong Kou District Health and Family Planning Commission Research Grants (No. 1503-31) and National Nature Science Foundation of China (No. 3870831). We also would like to thank all the contributors for the substantial information compiled in previously published papers that were cited in this manuscript. Their information was immensely helpful for preparing this manuscript.

\section{Conflict of Interest Statement}

The authors confirm no conflict of interest.

\section{References}

[1] Rosamond, W., Flegal, K., Friday, G., Furie, K., Go, A., Greenlund, K., et al. (2007) Heart Disease and Stroke Statistics-2007 Update: A Report from the American Heart Association Statistics Committee and Stroke Statistics Subcommittee. Circulation, 115, e69-e171. https://doi.org/10.1161/circulationaha.106.179918

[2] MacKay, J. and Mensah, G. (2004) The Atlas of Heart Disease and Stroke. World Health Organization.

[3] Mattila, K.J., Asikainen, S., Wolf, J., Jousimies-Somer, H., Valtonen, V. and Nieminen, M. (2000) Age, Dental Infections, and Coronary Heart Disease. Journal of Dental Research, 79, 756-760. https://doi.org/10.1177/00220345000790020901

[4] Emingil, G., Buduneli, E., Aliyev, A. and Atilla, G. (2000) Association between Periodontal Disease and Acute Myocardial Infarction. Journal of Periodontology, 71, 1882-1886. https://doi.org/10.1902/jop.2000.71.12.1882

[5] Ouyang, X.Y., Xiao, W.M., Chu, Y. and Zhou, S.Y. (2011) Influence of Periodontal Intervention Therapy on Risk of Cardiovascular Disease. Periodontology 2000, 56, 227-257. https://doi.org/10.1111/j.1600-0757.2010.00368.x

[6] Geerts, S.O., Legrand, V., Charpentier, J., Albert, A. and Rompen, E.H. (2004) Further Evidence of the Association between Periodontal Conditions and Coronary Artery Disease. Journal of Periodontology, 75, 1274-1280. https://doi.org/10.1902/jop.2004.75.9.1274

[7] Hung, H.C., Joshipura, K.J., Colditz, G., Manson, J.E., Rimm, E.B, Speizer, F.E., et al. (2004) The Association between Tooth Loss and Coronary Heart Disease in Men and Women. Journal of Public Health Dentistry, 64, 209-215. https://doi.org/10.1111/j.1752-7325.2004.tb02755.x

[8] Wu, T.J., Trevisan, M., Genco, R.J., Falkner, K.L., Dorn, J.P. and Christopher, T. (2000) Examination of the Relation between Periodontal Health Status and Cardiovascular Risk Factors: Serum Total and High Density Lipoproten Cholesterol, C-Reactive Protein, and Plasma Fibrinogen. American Journal of Epidemiology, 151, 273-282. https://doi.org/10.1093/oxfordjournals.aje.a010203

[9] Andriankaja, O.M., Genco, R.J., Dorn, J., Dmochowski, J., Hovey, K. and Falkner, K.L. (2007) Periodontal Disease and Risk of Myocardial Infarction: The Role of Gender and Smoking. European Journal of Epidemiology, 22, 699-705. https://doi.org/10.1007/s10654-007-9166-6

[10] Guntheroth, W.G. (1984) How Important Are Dental Procedures as a Cause of Infective Endocarditis. The American Journal of Cardiology, 54, 797-801. 
https://doi.org/10.1016/S0002-9149(84)80211-8

[11] Bahekar, A.A., Singh, S., Saha, S., Molnar, J. and Arora, R. (2007) The Prevalence and Incidence of Coronary Heart Disease Is Significantly Increased in Periodontitis: A Meta-Analysis. American Heart Journal, 154, 830-837. https://doi.org/10.1016/j.ahj.2007.06.037

[12] Brodala, N.M.E., Bellinger, D.A., Damrongsri, D. Offenbacher, S. and Beck, J. (2005) Porphyromonas gingivalis Bacteremia Induces Coronary and Aortic Atherosclerosis in Normocholesterolemic and Hypercholesterolemic Pigs. Arteriosclerosis, Thrombosis, and Vascular Biology, 25, 1446-1451. https://doi.org/10.1161/01.ATV.0000167525.69400.9c

[13] Meurman, J.H., Sanz, M. and Janket, S.J. (2004) Oral Health, Atherosclerosis and Cardiovascular Disease. Critical Reviews in Oral Biology \& Medicine, 15, 403-413. https://doi.org/10.1177/154411130401500606

[14] Persson, G.R. and Persson, R.E. (2008) Cardiovascular Disease and Periodontitis: An Update on the Associations and Risk. Journal of Clinical Periodontology, 35, 362-379. https://doi.org/10.1111/j.1600-051X.2008.01281.x

[15] Ioannidou, E., Malekzadeh, T. and Dongari-Bagtzoglou, A. (2006) Effect of Periodontal Treatment on Serum C-Reactive Protein Levels: A Systematic Review and Meta-Analysis. Journal of Periodontology, 77, 1635-1642. https://doi.org/10.1902/jop.2006.050443

[16] Roberts, G.J., Gardner, P. and Simmons, N.A. (1992) Optimum Sampling Time for Detection of Dental Bacteraemia in Children. International Journal of Cardiology, 35, 311-315. https://doi.org/10.1016/0167-5273(92)90228-U

[17] Strom, B.L., Abrutyn, E., Berlin, J.A., Kinman, J.L., Feldman, R.S., Stolley, P.D., et al. (1998) Dental and Cardiac Risk Factors for Infective Endocarditis. A Population-Based, CaseControl Study. Annals of Internal Medicine, 129, 761-769. https://doi.org/10.7326/0003-4819-129-10-199811150-00002

[18] Tzukert, A.A., Leviner, E. and Sela, M. (1986a) Prevention of Infective Endocarditis: Not by Antibiotics Alone. A 7-Year Follow-Up of 90 Dental Patients. Oral Surgery Oral Medicine and Oral Pathology, 62, 385-388.

[19] Seymour, R.A., Preshaw, P.M., Thomason, J.M., Ellis, J.S. and Steele, J.G. (2003) Cardiovascular Diseases and Periodontology. Journal of Clinical Periodontology, 30, 279-292. https://doi.org/10.1034/j.1600-051X.2003.00291.x

[20] Hanatani, T., Suzuki, J. and Ogawa, M. (2012) The Periodontal Pathogen Aggregatibacter actinomycetemcomitans Deteriorates Ventricular Remodeling after Myocardial Infarction in Mice. International Heart Journal, 53, 253-256. https://doi.org/10.1536/ihj.53.253

[21] Iwai, T. (2009) Periodontal Bacteremia and Various Vascular Diseases. Journal of Periodontal Research, 44, 689-694. https://doi.org/10.1111/j.1600-0765.2008.01165.x

[22] Haraszthy, V.I., Zambon, J.J., Trevisan, M., Zeid, M. and Genco, R.J. (2000) Identification of Periodontal Pathogens in Atheromatous Plaques. Journal of Periodontology, 71, 15541560. https://doi.org/10.1902/jop.2000.71.10.1554

[23] Kebschull, M., Demmer, R.T. and Papapanou, P.N. (2010) Gum Bug, Leave My Heart Alone! Epidemiologic and Mechanistic Evidence Linking Periodontal Infections and Atherosclerosis. Journal of Dental Research, 89, 879-902. https://doi.org/10.1177/0022034510375281

[24] Gaetti-Jardim, E., Marcelino, S.L., Feitosa, A.C.R., Romito, G.A. and Avila-Campos, M.J. (2009) Quantitative Detection of Periodontopathic Bacteria in Atherosclerotic Plaques from Coronary Arteries. Journal of Medical Microbiology, 58, 1568-1575. https://doi.org/10.1099/jmm.0.013383-0

[25] Mattila, K., Nieminen, M., Valtonen, V., Rasi, V., Kesaniemi, Y. and Syrjala, S. (1989) Association between Dental Health and Acute Myocardial Infarction. British Medical Journal, 298, 779-782. https://doi.org/10.1136/bmj.298.6676.779 
[26] Seymour, G.J. and Gemmell, E. (2001) Cytokines in Periodontal Disease: Where to from Here. Acta Odontologica Scandinavica, 59, 167-173. https://doi.org/10.1080/000163501750266765

[27] Nakajima, T., Honda, T. and Domon, H. (2010) Periodontitis-Associated Up-Regulation of Systemic Inflammatory Mediator Level May Increase the Risk of Coronary Heart Disease. Journal of Periodontal Research, 45, 116-122. https://doi.org/10.1111/j.1600-0765.2009.01209.x

[28] Boyce, B.F., Li, P., Yao, Z., Zhang, Q., Badell, I.R., Schwarz, E.M., et al. (2005) TNF-Alpha and Pathologic Bone Resorption. The Keio Journal of Medicine, 54, 127-131. https://doi.org/10.2302/kjm.54.127

[29] Abeywardena, M.Y., Leifert, W.R., Warnes, K.E., Varghese, J.N. and Head, R.J. (2009) Cardiovascular Biology of Interleukin-6. Current Pharmaceutical Design, 15, 1809-1821. https://doi.org/10.2174/138161209788186290

[30] Goncalves, T.O., Costa, D., Brodskyn, C.I., et al. (2010) Release of Cytokines by Stimulated Peripheral Blood Mononuclear Cells in Chronic Periodontitis. Archives of Oral Biology, 55, 975-980. https://doi.org/10.1016/j.archoralbio.2010.08.002

[31] Ridker, P.M., Rifai, N., Stampfer, M.J. and Hennekens, C.H. (2000) Plasma Concentration of Interleukin-6 and the Risk of Future Myocardial Infarction among Apparently Healthy Men. Circulation, 101, 1767-1772. https://doi.org/10.1161/01.CIR.101.15.1767

[32] Loos, B.G., Craandijk, J., Hoek, F.J., Wertheim-van, D.P.M. and van der, V.U. (2000) Elevation of Systemic Markers Related to Cardiovascular Diseases in the Peripheral Blood of Periodontitis Patients. Journal of Periodontology, 71, 1528-1534.

https://doi.org/10.1902/jop.2000.71.10.1528

[33] Higashi, Y., Goto, C., Hidaka, T., Soga, J., Nakamura, S., Fujii, Y., et al. (2009) Oral Infection-Inflammatory Pathway, Periodontitis, Is a Risk Factor for Endothelial Dysfunction in Patients with Coronary Artery Disease. Atherosclerosis, 206, 604-610. https://doi.org/10.1016/j.atherosclerosis.2009.03.037

[34] Czerniuk, M.R., Gorska, R., Filipiak, K.J. and Opolski, G. (2004) Inflammatory Response to Acute Coronary Syndrome in Patients with Coexistent Periodontal Disease. Journal of Periodontology, 75, 1020-1026. https://doi.org/10.1902/jop.2004.75.7.1020

[35] Go'rska, R., Gregorek, H., Kowalski, J., Laskus-Perendyk, A. and Syczewska, M. (2003) Relationship between Clinical Parameters and Cytokine Profiles in Inflamed Gingival Tissue and Serum Samples from Patients with Chronic Periodontitis. Journal of Clinical Periodontology, 30, 1046-1052. https://doi.org/10.1046/j.0303-6979.2003.00425.x

[36] Maynard, C.L. and Weaver, C.T. (2008) Diversity in the Contribution of Interleukin-10 to T Cell Mediated Immune Regulation. Immunological Reviews, 226, 219-233. https://doi.org/10.1111/j.1600-065X.2008.00711.X

[37] Buhlin, K., Hultin, M. and Norderyd, O. (2009) Periodontal Treatment Influences Risk Markers for Atherosclerosis in Patients with Severe Periodontitis. Atherosclerosis, 206, 518522. https://doi.org/10.1016/j.atherosclerosis.2009.03.035

[38] Yamazaki, K., Honda, T., Oda, T., Ueki-Maruyama, K., Nakajima, T., Yoshie, H., et al. (2005) Effect of Periodontal Treatment on the C-Reactive Protein and Proinflammatory Cytokine Levels in Japanese Periodontitis Patients. Journal of Periodontal Research, 40, 5358. https://doi.org/10.1111/j.1600-0765.2004.00772.x

[39] D’Aiuto, F., Parkar, M., Andreou, G., Suvan, J., Brett, P.M., Ready, D., et al. (2004) Periodontitis and Systemic Inflammation: Control of the Local Infection Is Associated with a Reduction in Serum Inflammatory Markers. Journal of Dental Research, 83, 156-160. https://doi.org/10.1177/154405910408300214

[40] Iwamoto,Y., Nishimura, F., Soga, Y., Takeuchi, K., Kurihara, M., Takashiba, S., et al. (2003) Antimicrobial Periodontal Treatment Decreases Serum C-Reactive Protein, Tumor Necro- 
sis Factor-Alpha, but Not Adiponection Levels in Patients with Chronic Periodontitis. Journal of Periodontology, 74, 1231-1236. https://doi.org/10.1902/jop.2003.74.8.1231

[41] Forner, L., Neilson, C.H., Bendtzen, K., Larsen, T. and Holmstrup, P. (2006) Increased Plasma Levels of IL-6 in Bacteremic Periodontis Patients after Scaling. Journal of Clinical Periodontology, 33, 724-729. https://doi.org/10.1111/j.1600-051X.2006.00964.x

[42] Black, S., Kushner, I. and Samols, D. (2004) C-Reactive Protein. The Journal of Biological Chemistry, 279, 48487-48490. https://doi.org/10.1074/jbc.R400025200

[43] Kaptoge, S., Di Angelantonio, E. and Lowe, G. (2010) C-Reactive Protein Concentration and Risk of Coronary Heart Disease, Stroke, and Mortality: An Individual Participant Meta-Analysis. The Lancet, 375, 132-140. https://doi.org/10.1016/S0140-6736(09)61717-7

[44] Ridker, P.M., Hennekens, C.H., Buring, J.E. and Rifai, N. (2000) C-Reactive Protein and Other Markers of Inflammation in the Prediction of Cardiovascular Disease in Women.

The New England Journal of Medicine, 342, 836-843. https://doi.org/10.1056/NEJM200003233421202

[45] Pasceri, V., Willerson, J.T. and Yeh, E.T. (2000) Direct Proinflammatory Effect of C-Reactive Protein on Human Endothelial Cells. Circulation, 102, 2165-2168. https://doi.org/10.1161/01.CIR.102.18.2165

[46] Sun, X.J., Meng, H.X., Shi, D., Xu, L., Zhang, L., Chen, Z.B., et al. (2009) Elevation of CReactive Protein and Interleukin-6 in Plasma of Patients with Aggressive Periodontitis. Journal of Periodontal Research, 44, 311-316. https://doi.org/10.1111/j.1600-0765.2008.01131.x

[47] Paraskevas, S., Huizinga, J.D. and Loos, B.G. (2008) A Systematic Review and Meta-Analyses on C-Reactive Protein in Relation to Periodontitis. Journal of Clinical Periodontology, 35, 277-290. https://doi.org/10.1111/j.1600-051X.2007.01173.x

[48] Tuter, G., Kurtis, B. and Serdar, M. (2007) Evaluation of Gingival Crevicular Fluid and Serum Levels of High-Sensitivity C-Reactive Protein in Chronic Periodontitis Patients with or without Coronary Artery Disease. Journal of Periodontology, 78, 2319-2324. https://doi.org/10.1902/jop.2007.070150

[49] Thakare, K.S., Deo, V. and Bhongade, M.L. (2010) Evaluation of the C-Reactive Protein Serum Levels in Periodontitis Patients with or without Atherosclerosis. Indian Journal of Dental Research, 21, 326-329. https://doi.org/10.4103/0970-9290.70787

[50] Vidal, F., Figueredo, C.M., Cordovil, I. and Fischer, R.G. (2009) Periodontal Therapy Reducesplasma Levels of Interleukin-6, C-Reactive Protein, and Fibrinogen in Patients with Severe Periodontitis and Refractory Arterial Hypertension. Journal of Periodontology, 80, 786-791. https://doi.org/10.1902/jop.2009.080471

[51] Offenbacher, S., Beck, J.D. and Moss, K. (2009) Results from the Periodontitis and Vascular Events (PAVE) Study: A Pilot Multi-Centered, Randomized, Controlled Trial to Study Effects of Periodontal Therapy in a Secondary Prevention Model of Cardiovascular Disease. Journal of Periodontology, 80, 190-201. https://doi.org/10.1902/jop.2009.080007

[52] Taylor, B., Tofler, G. and Morel-Kopp, M.C. (2010) The Effect of Initial Treatment of Periodontitis on Systemic Markers of Inflammation and Cardiovascular Risk: A Randomized Controlled Trial. European Journal of Oral Sciences, 118, 350-356.

https://doi.org/10.1111/j.1600-0722.2010.00748.x

[53] Acharya, A., Bhavsar, N., Jadav, B. and Parikh, H. (2010) Cardioprotective: Effect of Periodontal Therapy in Metabolic Syndrome: A Pilot Study in Indian Subjects. Metabolic Syndrome and Related Disorders, 8, 335-341. https://doi.org/10.1089/met.2010.0002

[54] D’Aiuto, F., Nibali, L., Parkar, M., Suvan, J. and Tonetti, M.S. (2005) Short-Term Effects of Intensive Periodontal Therapy on Serum Inflammatory Markers and Cholesterol. Journal of Dental Research, 84, 269-273. https://doi.org/10.1177/154405910508400312

[55] Izumi, Y., Nagasawa, T. and Umeda, M. (2009) Periodontitis and Cardiovascular Diseases: 
The Link and Relevant Mechanisms. Japanese Dental Science Review, 45, 98-108. https://doi.org/10.1016/j.jdsr.2009.06.001

[56] Hokamura, K. and Umemura, K. (2010) Roles of Oral Bacteria in Cardiovascular Diseases, from Molecular Mechanisms to Clinical Cases: Porphyromonas gingivalis is the Important Role of Intimal Hyperplasia in the Aorta. Journal of Pharmaceutical Sciences, 113, 110-114.

[57] Hall, G., Hedstrom, S.A., Heimdahl, A. and Nord, C.E. (1993) Prophylactic Administration of Penicillins for Endocarditis Does Not Reduce the Incidence of Postextraction Bacteremia. Clinical Infectious Diseases, 17, 188-194. https://doi.org/10.1093/clinids/17.2.188

[58] Macfarlane, T.W., Ferguson, M.M. and Mulgrew, C.J. (1984) Post-Extraction Bacteraemia: Role of Antiseptics and Antibiotics. British Dental Journal, 156, 179-181.

[59] Maharaj, B., Coovadia, Y. and Vayej, A.C. (2012) A Comparative Study of Amoxicillin, Clindamycin and Chlorhexidine in the Prevention of Post-Extraction Bacteraemia. Cardiovascular Journal of Africa, 23, 491-494. https://doi.org/10.5830/CVJA-2012-049

[60] Thornhill, M.H., Dayer, M.J., Forde, J.M., Corey, G.R., Chu, V.H., Couper, D.J., et al. (2011) Impact of the NICE Guideline Recommending Cessation of Antibiotic Prophylaxis for Prevention of Infective Endocarditis: Before and after Study. BMJ, 342, d2392. https://doi.org/10.1136/bmj.d2392

[61] Chen, T.L., Wang, S.F. and Zhang, X.H. (2012) Influence of Periodontitis and Nonsurgical Periodontal Intervention on Atherosclerosis Diseases. Advances in Bioscience and Biotechnology, 3, 531-537. https://doi.org/10.4236/abb.2012.324070

\section{Submit or recommend next manuscript to SCIRP and we will provide best service for you:}

Accepting pre-submission inquiries through Email, Facebook, LinkedIn, Twitter, etc. A wide selection of journals (inclusive of 9 subjects, more than 200 journals)

Providing 24-hour high-quality service

User-friendly online submission system

Fair and swift peer-review system

Efficient typesetting and proofreading procedure

Display of the result of downloads and visits, as well as the number of cited articles

Maximum dissemination of your research work

Submit your manuscript at: http://papersubmission.scirp.org/

Or contact ss@scirp.org 